\title{
Evaluation of Tinnitus and Hearing Loss in the Adult
}

\author{
Jenny K. Hoang and Laurie A. Loevner
}

\section{Learning Objectives}

- Identify key anatomical structures in the temporal bone.

- Describe role of CT and MRI for temporal bone imaging.

- Differentiate between diseases of the temporal bone on imaging and describe their clinical presentation.

\section{Key Points}

- Imaging can identify the cause and evaluate the extent of disease for surgical planning.

- The common causes of tinnitus and hearing loss without a mass include otospongiosis, labyrinthitis ossificans, superior semicircular canal dehiscence, and enlarged vestibular aqueduct syndrome.

- Otospongiosis affects the bony labyrinth, while labyrinthitis ossificans affects the membranous labyrinth.

- Vestibular schwannomas often present with nonpulsatile tinnitus and high frequency sensorineural hearing loss.

\subsection{Introduction}

Tinnitus and hearing loss in the adult can have profound effects on the quality of life. Tinnitus is the perception of sound when no external sound is present and may be described as ringing, buzzing, swishing, or clicking sensations. Hearing

J. K. Hoang $(\bowtie)$

Department of Radiology and Radiological Science,

Russell H. Morgan, Johns Hopkins School of Medicine,

Baltimore, MD, USA

\section{A. Loevner}

Department of Neuroradiology, Radiology, Neurosurgery,

Otorhinolaryngology: Head \& Neck Surgery, and Ophthalmology,

University of Pennsylvania, Philadelphia, PA, USA loss ranges from partial to a total inability to hear sounds. Hearing loss and tinnitus can occur concurrently or in isolation. The role of imaging is to help identify the etiology of these symptoms and evaluate for the extent of disease.

\subsection{Causes of Tinnitus and Hearing Loss}

\subsubsection{Tinnitus}

Tinnitus may be categorized as (1) pulsatile or non-pulsatile, (2) primary (idiopathic) or secondary (due to another condition), and (3) subjective or objective. Evaluation of tinnitus starts with otoscopic examination to determine if there is a vascular retro-tympanic mass, audiometric examination, and review of medical history and medications. This evaluation helps to determine if imaging is necessary and if so, what study(ies) are indicated.

\subsubsection{Pulsatile Tinnitus}

Causes include vascular masses (glomus tympanicum), aberrant arterial or venous anatomy, vascular malformations, and intracranial hypertension. Objective tinnitus (auscultation of a bruit on physical examination) is uncommon and has been attributed to turbulent flow in the setting of dural fistulas, atherosclerotic carotid artery disease, jugular bulb abnormalities, and large condylar or mastoid emissary veins.

\subsubsection{Non-pulsatile Tinnitus}

Causes are wide ranging and include cerumen impaction, middle ear infection, mass, medications, noise-induced hearing loss, presbycusis or chronic bilateral hearing loss, hemorrhage, neurodegeneration, and spontaneous intracranial hypotension.

\subsubsection{Hearing Loss}

Clinical assessment and audiometric testing can determine the type of hearing loss as conductive, sensorineural, or 
mixed and guide subsequent diagnostic imaging. Conductive hearing loss results from diseases affecting the conduction of mechanical sound wave energy to the cochlea. Sensorineural hearing loss is caused by diseases that impair the cochlear function or the transmission of electrical signal along the auditory pathway.

\subsubsection{Conductive Hearing Loss}

Causes include otospongiosis (commonly mixed), ossicular erosion or fusion, round window occlusion, dehiscence of the superior semicircular canal, and cholesteatoma or neoplasm with suspected intracranial or inner ear extension.

\subsubsection{Sensorineural Hearing Loss}

Causes include labyrinthine ossificans, vestibular schwannoma, and fractures extending across the otic capsule.

\subsection{Anatomy}

The temporal bone forms part of the middle and posterior cranial fossae and is comprised of five parts: petrous, tympanic, mastoid, styloid, and squamous temporal bone. The first three listed parts form the external auditory canal, middle ear, inner ear, and internal auditory canal. These are the segments to review for hearing loss and tinnitus.

\subsubsection{External Auditory Canal}

The external auditory canal (EAC) extends from the auricle to the tympanic membrane. The lateral one-third of the EAC is fibrocartilaginous, while the medial two-thirds are surrounded by the tympanic portion of the temporal bone.

\subsubsection{Middle Ear}

The middle ear cavity contains the ossicular chain which conducts sound from the tympanic membrane laterally, to the oval window and inner ear structures medially. The roof of the middle ear is the tegmen tympani and the jugular wall is the floor. The middle ear can be subdivided into the epitympanum (attic) superior to the level of the tympanic membrane, mesotympanum at the level of tympanic membrane, and hypotympanum inferior to the level of tympanic membrane. The epitympanum communicates with the mastoid via the aditus ad antrum.

The mesotympanum contains the majority of the ossicular chain. The ossicular chain is composed of three bones: the malleus, incus, and stapes. Since the stapes is anchored to the oval window, a mnemonic for the order of the ossicles is "MISO" representing malleus, incus, stapes, and oval win- dow. The manubrium of the malleus is attached to the tympanic membrane, and the head of the malleus articulates with the body of the incus in the epitympanum forming the incudomalleal joint, which has a characteristic "ice cream cone" configuration on axial sections. The lenticular process of the incus extends at approximately a right angle from the long process of the incus to articulate with the capitulum (head) of the stapes, forming the incudostapedial joint.

An important middle ear structure is the scutum, a sharp bony projection to which the tympanic membrane is attached superiorly. Prussak space, the location for pars flaccida cholesteatomas, is bounded by the scutum laterally and the neck of the malleus medially.

The posterior wall of the middle ear is irregular and includes the sinus tympani, pyramidal eminence, and facial recess.

\subsubsection{Inner Ear}

The inner ear comprises the osseous labyrinth, which includes the cochlea, vestibule, and semicircular canals. The cochlea contains the end organ for hearing while the vestibule and semicircular canals are responsible for balance and equilibrium. The densest portion of the temporal bone that surrounds the osseous labyrinth is termed the otic capsule. The osseous labyrinth encapsulates the membranous labyrinth, which contains endolymph and is surrounded by perilymph.

The cochlea is a spiral-shaped structure with $2 \frac{1}{2}-2 \frac{3}{4}$ turns, including the basal, middle, and apical turns, which are separated by interscalar septa. The lateral aspect of the basal turn of the cochlea bulges into the middle ear cavity, forming the cochlear promontory. The nerve of Jacobson (branch of cranial nerve IX) courses over the cochlear promontory. The cochlear nerve passes from the internal auditory canal through the bony canal for the cochlear nerve (also referred to as the cochlear fossette or cochlear aperture) into the modiolus, which is a crown-shaped structure centered within the cochlea that transmits branches of the cochlear nerve to the organ of Corti. The organ of Corti is the end organ for hearing and is not visible on CT images.

The bony vestibule is an ovoid space located superior and posterior to the cochlea, which connects to the semicircular canals. There are three semicircular canals-superior, posterior, and lateral which are oriented orthogonal to one another. The endolymphatic duct extends from the posterior aspect of the vestibule toward the posterior cranial fossa, and ends in a blind pouch, the endolymphatic sac, at the posterior margin of the petrous ridge. The bony vestibular aqueduct surrounds the endolymphatic duct and normally measures up to $1 \mathrm{~mm}$ at the midpoint and $2 \mathrm{~mm}$ at the operculum, according to the Cincinnati criteria [1]. 
The cochlear aqueduct should not be mistaken for a fracture. It is a narrow bony channel that surrounds the perilymphatic duct and extends from the basal turn of the cochlea to the subarachnoid space adjacent to the pars nervosa of the jugular foramen.

\subsubsection{Internal Auditory Canal}

The internal auditory canal (IAC) is a channel in the petrous bone. It extends from the fundus which abuts the labyrinth to the porus acusticus. At the fundus, a transverse crest (crista falciformis) divides the IAC into superior and inferior compartments. A vertical crest ("Bill's bar") divides the superior compartment into anterior and posterior components. The facial nerve is located in the anterosuperior compartment, the cochlear nerve in the anteroinferior compartment, and the superior and inferior vestibular nerves in the superoposterior and inferoposterior compartments, respectively. A mnemonic for the location of the nerves in the anterior compartment is "Seven (cranial nerve VII) Up Coke (cochlear nerve) down."

\subsection{Imaging Modalities and Techniques}

While CT is often the first modality utilized to assess suspected pathology involving the inner ear structures (cochlea and labyrinth), certain lesions such as a cochlear schwannoma and labyrinthine hemorrhage are better detected with MRI. In the work-up of subjective pulsatile tinnitus, CT is frequently the preferred modality. However, MR imaging with MRA and MRV may be more appropriate in the workup of objective pulsatile tinnitus (audible bruit on auscultation by the clinician). Conventional catheter angiography may then follow to further characterize the vascular abnormality as well as to provide therapeutic management (embolization) of lesions such as dural fistulas. MR imaging is the primary modality for evaluating the non-osseous components of the temporal bone, suspected retrocochlear pathology, and sensorineural hearing loss. Magnetic resonance imaging is required when temporal bone pathology is suspected to involve the intracranial compartment.

\subsubsection{Computed Tomography}

\subsubsection{Temporal Bone CT Technique}

Dual acquisition temporal bone CT (separately acquired direct axial and direct coronal images) has largely been replaced with multidetector row CT (MDCT) in which a single set/volume of axial images are acquired and reformatted in multiple planes. Thoughtfully performed MDCT reduces radiation dose, and its rapid acquisition minimizes artifact from patient motion. Intravenous contrast is typically not used with temporal bone CT because when contrast is necessary (tumors, vascular pathology such as dural fistulas), then higher resolution MRI, MRI with MRA, or CTA are usually obtained.

For temporal bone $\mathrm{CT}$ the patient is placed supine in the gantry with the head angled superiorly and posteriorly. The neck is hyperextended so that the orbits are canted out of the pathway of the X-ray beam to minimize exposure to the lens. Gantry tilt may need to be avoided to facilitate image reconstruction and reformats. Scan coverage is from the roof of the temporal bone (the arcuate eminence) through the mastoid tip. Thin collimation to achieve high resolution is extremely important as the anatomy of the temporal bone involves very small structures. A collimation of $0.6-1.0 \mathrm{~mm}$ provides appropriate resolution. The raw data from each ear is most often reconstructed into $0.6-0.75 \mathrm{~mm}$ thin axial images in bone algorithm at a DFOV of $100 \mathrm{~mm}$ that effectively magnifies the images. Technologists provide reconstructed images including $0.6-1.0 \mathrm{~mm}$ reformats in the axial plane parallel to the lateral semicircular canal created from a sagittal plane, and $0.6-1.0 \mathrm{~mm}$ reformats in the coronal plane. Axial images with $2-3 \mathrm{~mm}$ section thickness of the entire scan volume are also provided in soft tissue algorithm.

Reformats may be obtained in sagittal or oblique planes to improve the detection of pathology in specific clinical settings such as superior semicircular canal dehiscence.

Stenver reformat: On the console the technologist scrolls through the sagittal plane until a view of the lateral semicircular canal is obtained. A reformatted axial plane parallel to the lateral semicircular canal is made. The technologist then scrolls through this axial data set and Stenver reformats are made by tracing a line perpendicular to the long axis of the summit of the superior semicircular canal at submillimetric intervals. This plane is perpendicular to the roof of the superior semicircular canal displaying it in cross section.

Poschl reformat: On the console the technologist scrolls through the sagittal plane until a view of the lateral semicircular canal is obtained. A reformatted axial plane parallel to the lateral semicircular canal is made. The technologist then scrolls through this axial data set and Poschl reformats are made by tracing a line parallel to the long axis of the summit of the superior semicircular canal at submillimetric intervals.

\subsubsection{Magnetic Resonance Imaging}

MRI of the temporal bones is performed in a head coil and should include thin section unenhanced and enhanced axial T1-weighted images, and enhanced coronal T1-weighted 
images. Fat suppression in at least one enhanced plane should be performed to distinguish fat from enhancement, especially in the region of the petrous apex. A maximum section thickness of $3 \mathrm{~mm}$ with no interslice gap and a small FOV are required to provide images able to depict the fine detail of the anatomy and pathology in the temporal bone. Thin section (1 $\mathrm{mm}$ or submillimetric), 3D T2-weighted techniques are valuable in temporal bone imaging to evaluate the relationship of pathologic processes such as a vestibular schwannoma with the surrounding nerves, the patency of labyrinthine structures, the size of the endolymphatic duct and sac, and the extent of cochlear dysplasia in congenital or developmental hearing loss. Reformatted oblique sagittal images perpendicular to the long axis of the internal auditory canal are helpful in identifying cranial nerve hypoplasia or aplasia. Diffusion-weighted imaging (DWI) can help in distinguishing cholesteatoma which typically have restricted diffusion from inflammatory middle ear cavity opacification which has facilitated diffusion.

Axial T2-weighted, flair, and enhanced T1-weighted images of the brain should be performed to assess for intracranial extension of temporal bone pathology, and also to exclude central nervous system pathology that might produce tinnitus, vertigo, hearing loss, or cranial nerve symptoms such as demyelinating disease, stroke, or inflammation/ infection.

\subsection{Tinnitus and Hearing Loss without a Mass}

When no mass is seen on imaging, the radiologist should closely review the inner ear structures. The common causes of tinnitus and hearing loss without a mass include otospongiosis, labyrinthitis ossificans, superior semicircular canal dehiscence, and enlarged vestibular aqueduct syndrome.

\subsubsection{Otospongiosis}

Otospongiosis is an idiopathic progressive disease of pathological bone remodeling that causes conductive, sensorineural, or mixed hearing loss. It results in spongiosis or sclerosis of portions of the petrous bone. Conductive hearing loss is usually secondary to abnormal bone encroaching on stapes with impingement on the stapes footplate. Therefore, stapes surgery is the traditional initial treatment for otosclerosis, but is being augmented or replaced by innovations in hearing aid technology and cochlear implants.

Symptoms typically start in the second or third decade of life and presentation is with progressive conductive hearing loss ( $80 \%$ bilateral) with a normal tympanic membrane, and no evidence of middle ear inflammation. On otoscopic exam-

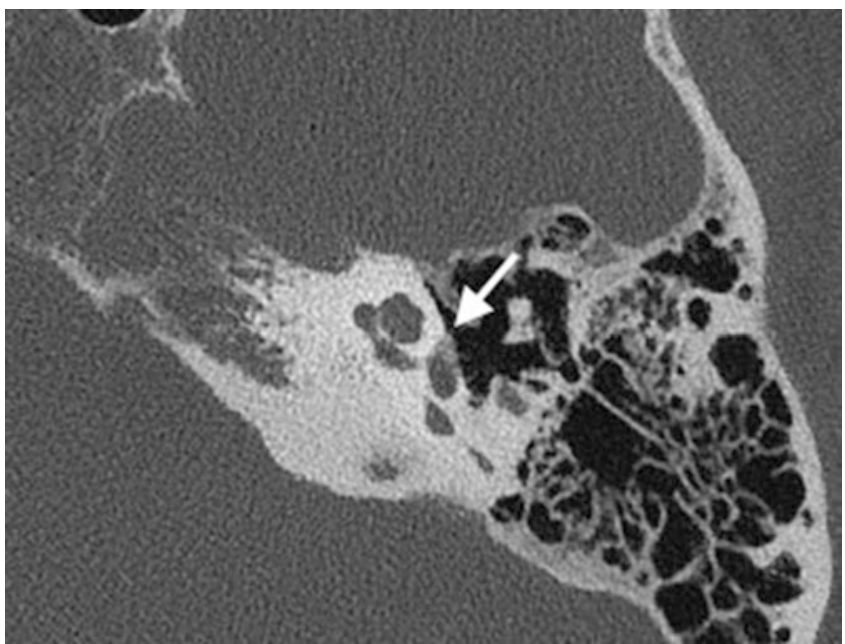

Fig. 15.1 Fenestral otospongiosis on the left with signs of lucency in the fissula ante fenestram (arrow)

ination, the promontory may have a faint pink tinge reflecting the vascularity of the lesion, referred to as the Schwartze sign.

On imaging, the disease is seen as lucency in the otic capsule. The most common location of involvement of otosclerosis is the bone just anterior to the oval window at a small cleft known as the fissula ante fenestram (fenestral otosclerosis) (Fig. 15.1). Involvement of the otic capsule separable from the oval window is referred to as "retrofenestral otosclerosis" or "cochlear otosclerosis" and can give a sensorineural component to the hearing loss.

A later presumably less active phase can occur where the bone becomes more dense and sclerotic. The bone still appears to encroach on the footplate but has a density closer to that of the otic capsule and thus may be difficult to identify.

\subsubsection{Labyrinthitis Ossificans}

Labyrinthitis ossificans is the late stage of labyrinthitis, in which there is pathologic ossification of spaces within the membranous labyrinth. Profound bilateral hearing loss from labyrinthitis ossificans is most commonly due to bacterial meningitis with onset of symptoms 3-4 months after the episode [2]. Other causes include trauma, hemorrhage, autoimmune disease, vascular obstruction of labyrinthine artery, and surgical insult.

Imaging can detect the evolution of labyrinthitis in three stages: acute, fibrous, and ossification. In the acute stage, enhancement of the inner ear is noted on MR images, but the CT scan may appear normal. In the intermediate fibrous stage of labyrinthitis, there is loss of fluid signal intensity on heavily T2-weighted sequence images (Fig. 15.2), while the 


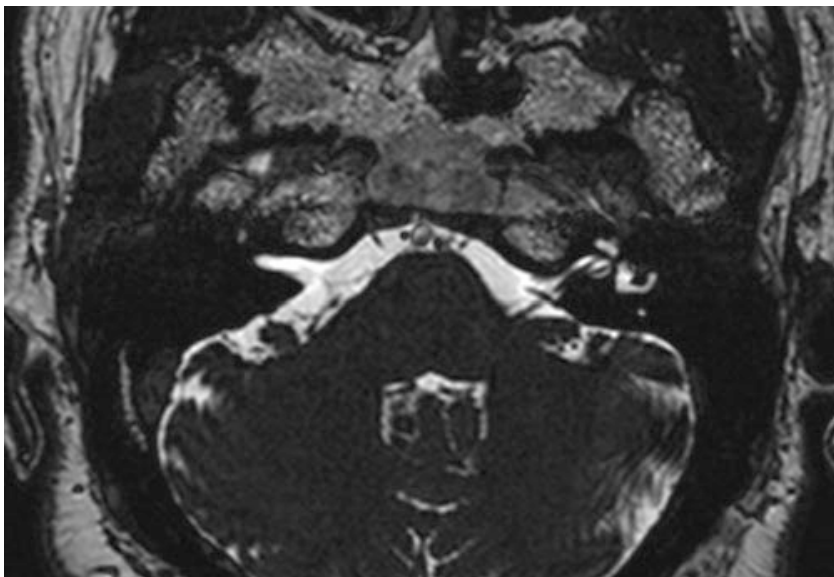

Fig. 15.2 Labyrinthitis ossificans on the right with loss of fluid signal intensity on heavily $\mathrm{T} 2$-weighted sequence images

CT scan may still appear normal. In the late ossific stage, one sees replacement of the normal cochlea, vestibule, and/or semicircular canals by bone attenuation on CT scans.

\subsubsection{Superior Semicircular Canal Dehiscence}

Superior semicircular canal dehiscence is the most common type of a group of disorders known as third window abnormalities. The labyrinth is normally in a closed hydraulic system with the oval and round windows. Defects in the integrity of the bony structure of the inner ear can decompress/dampen the energy of the sound wave resulting in conductive hearing loss.

In addition to conductive hearing loss, superior semicircular canal dehiscence can present with characteristic symptoms of vertigo when exposed to loud sounds (Tullio phenomenon). This is because of movement of fluid in the superior canal without movement in other canals. Other symptoms include autophony and pulsatile tinnitus. At audiometry, there is a characteristic low-frequency air-bone gap due to decreased air conduction and increased bone conduction.

On imaging, there is a defect in the superior semicircular canal, best seen in the coronal plane, and on reformatted oblique images in the Stenvers plane (perpendicular to the canal). The Pöschl plane shows the superior canal as a ring and helps to determine the length of a dehiscence (Fig. 15.3). The defect is typically along the superior arc of the superior canal along the floor of the middle cranial fossa. However, a defect can also occur along the posterior limb of the canal facing the posterior fossa.

Other third window abnormalities present with similar symptoms and include enlargement of the opening of the vestibular aqueduct, dehiscence of the scala vestibuli side of the cochlea, erosion of the lateral semicircular canal by cho-

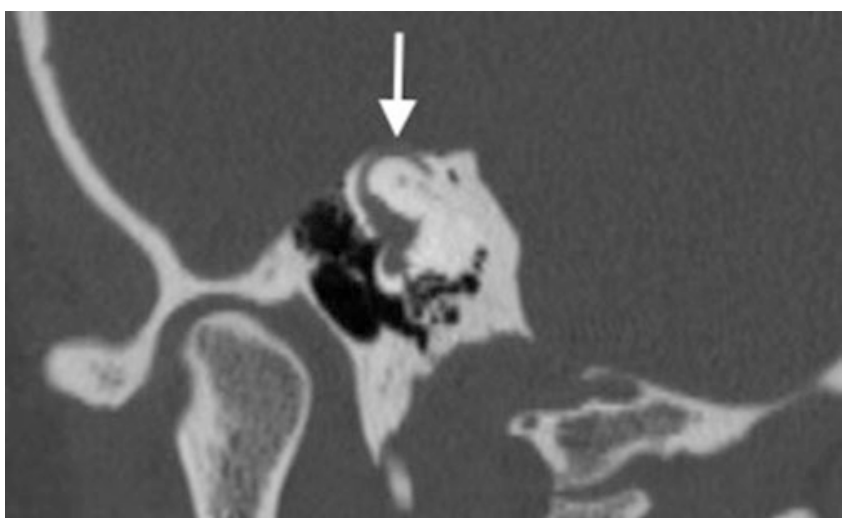

Fig. 15.3 Superior semicircular canal dehiscence on the left. There is a bone defect (arrow) in the superior semicircular canal. Pöschl plane shows the superior canal as a ring and helps to determine the length of a dehiscence

lesteatoma, and abnormal bony thinning between the cochlea and vascular channels. Presence of a defect does not mean that the patient will necessarily have symptoms or will benefit from surgery. Asymptomatic defects are rare (2\% of the population) so further audiometric evaluation is recommended for all patients with imaging findings [3].

\subsubsection{Enlarged Vestibular Aqueduct Syndrome}

Most congenital hearing malformations are detected after birth on newborn hearing screening. The exception is enlarged vestibular aqueduct syndrome (EVA) which can progress in childhood or less commonly early adulthood [4]. Stable hearing is observed in $67 \%$ of ears with EVA of which $34 \%$ will demonstrate fluctuations in hearing. Progression of hearing loss is seen in $33 \%$ of ears of which half will demonstrate fluctuations [5].

During fetal development, the vestibular aqueduct starts out as a wide tube. By the fifth week it narrows, and by midterm it approaches adult dimension and shape. However, the vestibular aqueduct continues to grow and change until a child is 3-4 years old. As yet incompletely understood genetic and/or environmental conditions cause EVA.

It is believed that an EVA does not cause hearing loss, but instead EVA and hearing loss are caused by the same underlying defect (gene mutations). Syndromic EVA includes Pendred syndrome or branchiootorenal syndrome. Syndromic hearing loss associated with EVA can affect other areas of the body. More commonly, the hearing loss associated with EVA is nonsyndromic, affecting only ear function.

On clinical exam, hearing loss can be conductive, mixed, or sensorineural, and the loss may be stable or fluctuating. Imaging is both diagnostic and prognostic (Fig. 15.4). There 


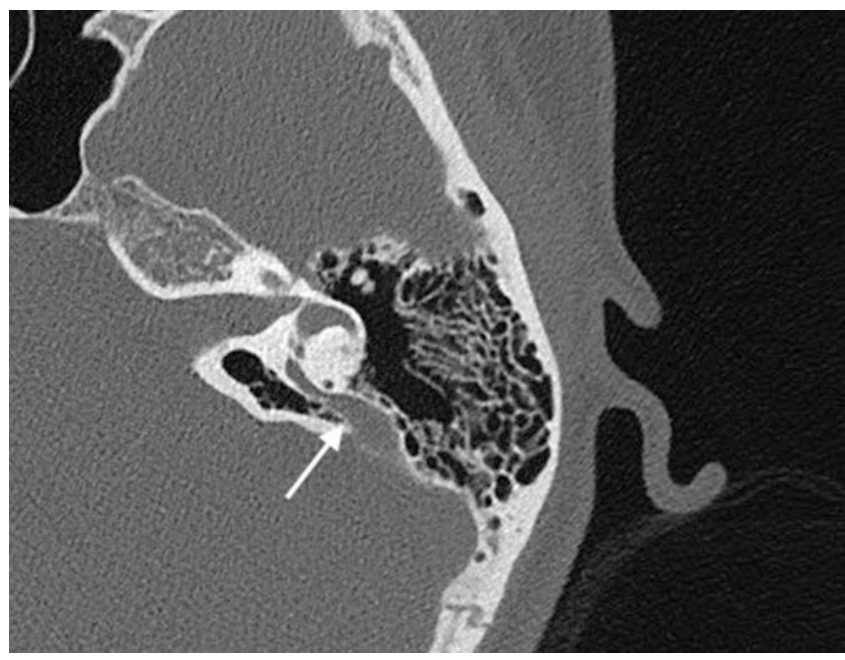

Fig. 15.4 Enlarged vestibular aqueduct. The vestibular aqueduct extends from the posterior aspect of the vestibule toward the posterior cranial fossa (arrow)

are several definitions of an enlarged vestibular aqueduct. The most sensitive criteria is the Cincinnati criteria which defines abnormal as $>0.9 \mathrm{~mm}$ at the midpoint or $>1.9 \mathrm{~mm}$ at the operculum in the axial view [1]. Other criteria are using the semicircular canal as reference-enlarged if width at the proximal intraosseous portion exceeded that of the adjacent posterior semicircular canal. A retrospective study has found the severity of hearing loss in patients with an EVA is influenced by degree of widening of the vestibular aqueduct midpoint width [6].

\subsection{Tinnitus and Hearing Loss with a Mass}

\subsubsection{Vestibular Schwannoma}

Vestibular schwannomas typically arise from perineural Schwann cells of the superior or inferior vestibular nerve in the internal auditory canal near the porus acusticus. They frequently extend into the cerebellopontine angle. Vestibular schwannomas often present with non-pulsatile tinnitus and high frequency sensorineural hearing loss. Less often, they may present with symptoms related to mass effect upon the middle cerebellar peduncle, lateral pons, and/or the cisternal trigeminal nerve. The presence of bilateral vestibular schwannomas is diagnostic of neurofibromatosis type II.

Vestibular schwannomas are typically difficult to identify on CT unless quite large. Expansion of the internal auditory canal may be present. On MR imaging, they are heterogeneous on T1- and T2-weighted images, and enhance following contrast administration. When large $(>2 \mathrm{~cm})$, they often show cystic degeneration. Five to $10 \%$ of vestibular schwannomas may have a co-existent arachnoid cyst. Hemorrhage may be seen in 5\%. Calcification is rare. When evaluating a patient with a vestibular schwannoma, it is important to identify the full extent of the neoplasm including extension to the cochlear aperture and/or cerebellopontine angle, and to describe the presence of mass effect on the middle cerebellar peduncle and brainstem. Approximately $60-75 \%$ of vestibular schwannomas involve the internal auditory canal and the cerebellopontine angle.

The goal of management of vestibular schwannomas over the last decade has shifted from "complete resection" to hearing preservation. Treatment may include surgical resection, stereotactic radiosurgery, and for small, stable tumors clinical and radiologic observation is now common [7].

\subsubsection{Cholesteatoma}

Cholesteatoma is an expansile erosive mass lined by keratinizing stratified squamous epithelium. It may result from a congenital inclusion of squamous epithelium in the middle ear cavity (congenital cholesteatoma), or more commonly from abnormal migration of squamous epithelium into the middle ear cavity through a perforated tympanic membrane as a result of chronic infection/inflammation. Otoscopic examination typically reveals a pearly white mass behind the tympanic membrane. Erosive enzymes and osteoclaststimulating agents within the epithelial debris result in bone destruction which is the radiologic hallmark of cholesteatomas. Acquired cholesteatomas most often occur in the setting of an under-pneumatized mastoid, a perforation of the pars flaccida (Shrapnell's membrane), involve Prussak space, and erode the adjacent bone including the scutum and ossicles (the mallear head and incus body). The ossicles may be displaced medially. Cholesteatomas related to pars tensa perforations are less common. In these lesions the sinus tympani, pyramidal eminence, and facial nerve recess may be involved and eroded. The long process of the incus and the stapes suprastructure may also be involved or eroded.

In the imaging evaluation of patients with a cholesteatoma, it is important to comment on the extent of middle ear cavity opacification. Specifically, it is important to note if there is involvement of the anterior epitympanic recess, round window niche, sinus tympani, and/or facial recess. The integrity of adjacent bone including the roof of the epitympanum (the tegmen tympani), the lateral semicircular canal, and the facial nerve canal should be commented upon. Involvement of important regional anatomy relevant to surgical planning such as the presence of high or dehiscent jugular bulb, or an anteriorly positioned sigmoid sinus plate should also be addressed in the radiologic interpretation. Diffusion-weighted MR imaging may be valuable in distinguishing cholesteatomas (which typically have restricted diffusion) from inflammatory opacification in the middle ear 
cavity. Frequently both are present, and DWI can distinguish the cholesteatoma form the areas of inflammation [8].

\subsubsection{Glomus Tumor}

When imaging the patient with a suspected glomus tympanicum, the radiologist should evaluate for and exclude other causes of pulsatile tinnitus including vascular anomalies such as an aberrant internal carotid artery, high riding dehiscent jugular bulb, jugular vein or sigmoid diverticulum (outpouching of the jugular bulb or sigmoid sinus, respectively, into adjacent pneumatized temporal bone), and large condylar or mastoid emissary veins that traverse air cells.

Glomus tympanicum is a paraganglioma that arises from glomus bodies along the course of Jacobson's nerve (the tympanic branch of CN IX) along the lateral aspect of the cochlea in the middle ear cavity. Glomus tympanicum is the most common primary tumor of the middle ear in adults, and presents in middle age with a slight female predominance. The typical clinical presentation is pulsatile tinnitus. Patients may also have conductive hearing loss. On otoscopic examination a fleshy vascular mass behind the tympanic membrane in the middle ear cavity is most often identified.

The classic imaging appearance is a demarcated mass along the cochlear promontory (Fig. 15.5). These tumors are soft tissue in density on $\mathrm{CT}$, and on MR imaging they are iso- to mildly hyperintense to CSF on T1-weighted imaging, mildly hyperintense on T2-weighted imaging, and enhance intensely following contrast administration [9]. While these tumors are very vascular, the typical "salt and pepper" appearance seen in other glomus tumors/paragangliomas is frequently not seen in glomus tympanicum tumors due to their relatively small size. Once establishing the presence of

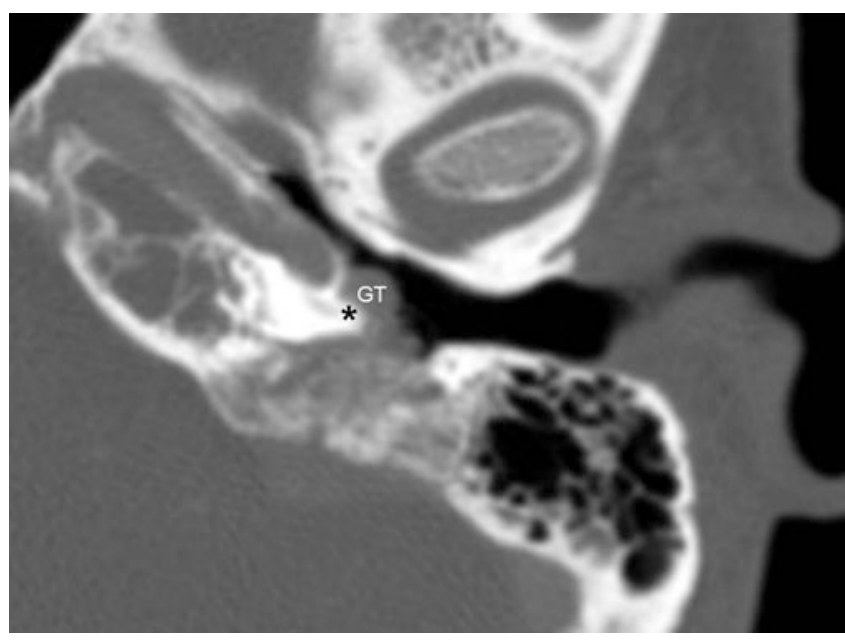

Fig. 15.5 Glomus tympanicum. Axial multidetector CT image of the left temporal bone shows a soft tissue mass (GT) at the cochlear promontory (asterisk) a glomus tympanicum it is important to determine the extent of disease. These tumors are classified based on their spread from the cochlear promontory to the middle ear cavity/ossicles, inner ear structures, external auditory canal, carotid canal, and the mastoid air cells. The radiologist should specify if the tumor is confined to the middle ear cavity (glomus tumors tend to engulf rather than erode the ossicles), identify if there is fistulization to the inner ears structures due to bone erosion, and identify extension of tumor into adjacent structures.

When there is tumor in the jugular foramen it is important to establish if the middle ear tumor is a projection of a larger glomus tumor arising in the jugular fossa-a glomus jugulare-tympanicum. Glomus jugulare paragangliomas arise from glomus bodies in the jugular foramen, either in the adventitia of the jugular bulb, the superior ganglion of cranial nerve CN X, or along Arnold or Jacobsen nerves (auricular branch of $\mathrm{CN} \mathrm{X}$ and tympanic branch of $\mathrm{CN}$ IX, respectively). They frequently grow directly into the jugular vein (Fig. 15.6a, b).

\subsubsection{Cholesterol Granuloma}

Lucent, expansile lesions of the petrous apex (cholesterol granuloma, mucocele, epidermoid, meningocele) are common. They may be incidental lesions detected on CT of the head being performed for unrelated reasons, or they may be identified on a head CT or a temporal bone CT performed for related symptomatology. These lesions may cause symptoms due to mass effect on adjacent structures. The management of each petrous apex lesion is different and clinical history is often of limited use. Biopsy is often difficult to perform, and in certain instances are contraindicated (meningocele) and potentially harmful to the patient. Fortunately, a specific diagnosis can usually be made using a combination of CT and MR imaging together $[10,11]$. On CT the pattern of bone expansion, remodeling, and/or erosion are important. On MR imaging the signal intensity on T1, T2, flair, DWI and enhanced T1 weighting are often diagnostic (e.g., cholesterol granulomas are typically hyperintense on unenhanced T1-weighted imaging, epidermoids classically have restricted diffusion) $[10,11]$. The size, location of the lesion, and its relationship to adjacent critical structures including the petrous internal carotid artery and the internal auditory canal are important to the referring physician.

Cholesterol granulomas typically arise in a pneumatized space, most often the petrous apex of the temporal bone. These develop due to recurrent micro-hemorrhages at the capillary level. This may be due to negative pressures in petrous air cells. The blood incites a mucosal reaction, giant cells, and cholesterol crystal deposition leading to recurrent hemorrhage. The cycle results in petrous apex 

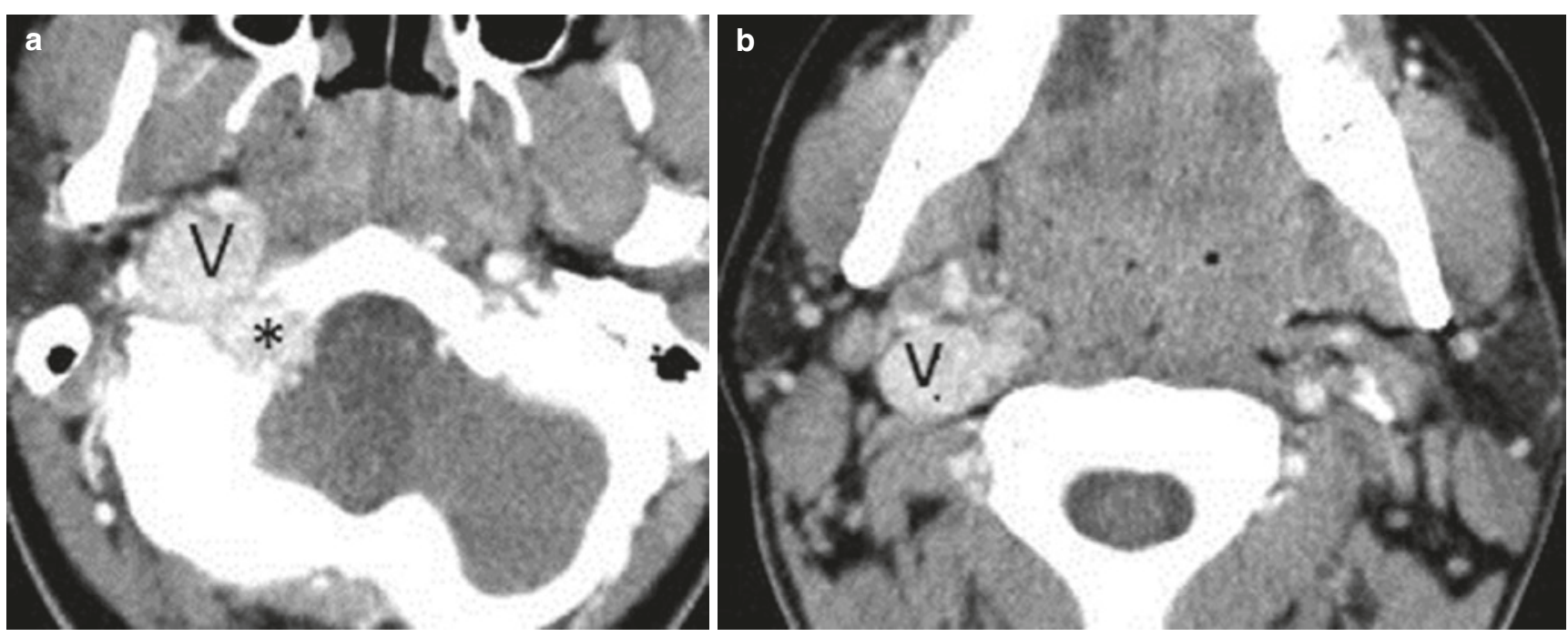

Fig. 15.6 (a, b). Glomus jugulare. Axial enhanced CT images show a vascular avidly enhancing mass in the jugular foramen (*), with direct inferior growth into the internal jugular vein $(\mathrm{V})$

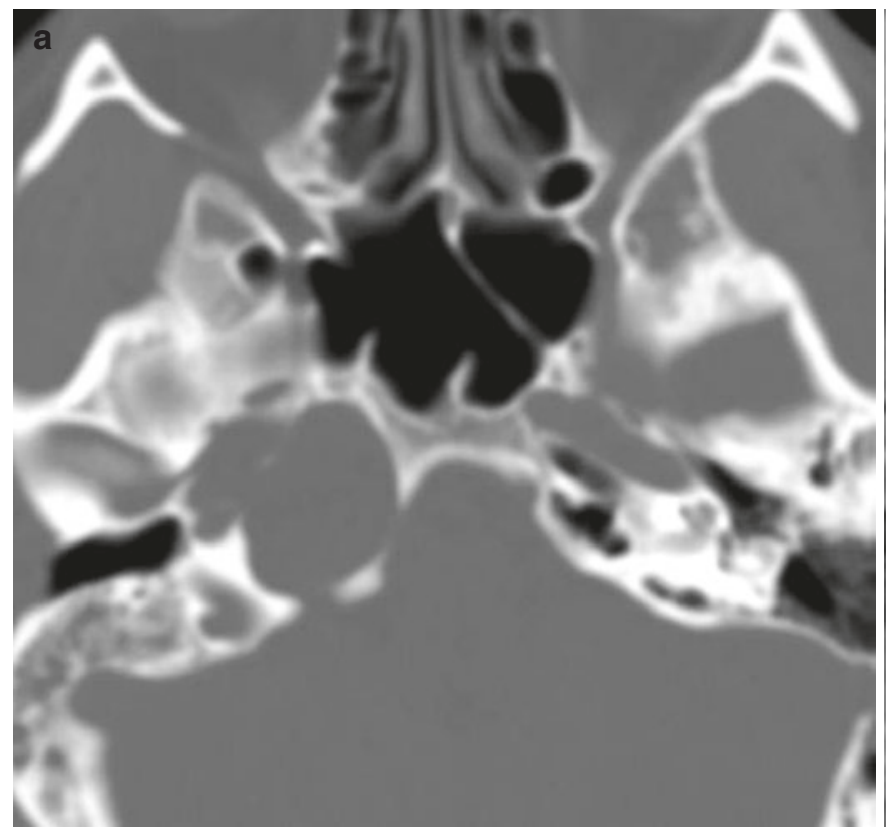

Fig. 15.7 (a, b). Cholesterol granuloma. (a) Axial CT image in bone window shows a unilocular, expansile mass of the right petrous apex. (b) Axial unenhanced T1-weighted gradient image shows the character-

expansion. This can result in a clinical presentation with cranial nerve symptoms (CNs V and VIII) and tinnitus. Cholesterol granulomas are expansile, lytic, circumscribed masses centered in the petrous apex and they are often unilocular (Fig. 15.7a). On MR imaging, cholesterol granulomas while heterogeneous on all pulse sequences due to blood products of varying ages, they are characteristically hyperintense on T1-weighted images related to methemoglobin (Fig. 15.7b).

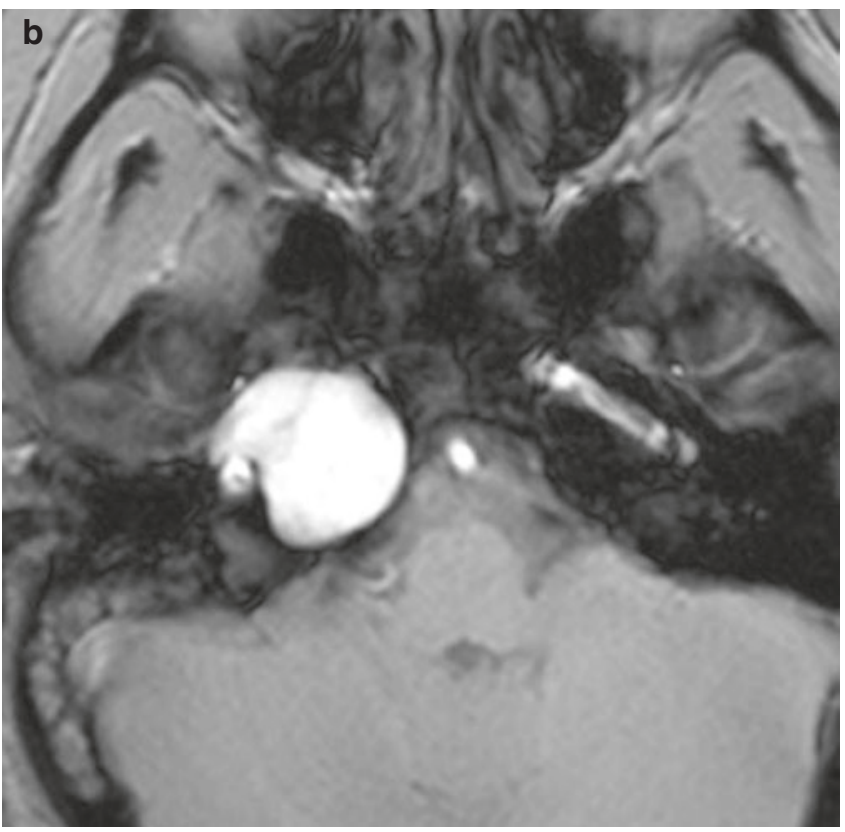

istic hyperintensity of these lesions related to blood products. The right mastoid air cells are opacified

\subsection{Conclusion}

The imaging workup for tinnitus and hearing loss in adults follows otoscopic exam and audiometry testing. Computed tomography and MR imaging have different and often complementary roles in the evaluation of tinnitus and hearing loss depending on the clinical scenario and the suspected underlying cause. Imaging can often identify the cause, and evaluate the extent of disease for surgical planning. 


\section{Take Home Messages}

- The radiologist should be familiar with a few key temporal bone anatomical structures and levels on axial and coronal imaging.

- Imaging approach and differentials for hearing loss and tinnitus can be based on whether a mass is present or absent.

- MR and CT are complementary modalities. The choice of imaging depends on the most likely clinical diagnosis following history and otoscopic examination.

\section{References}

1. El-Badry MM, Osman NM, Mohamed HM, Rafaat FM. Evaluation of the radiological criteria to diagnose large vestibular aqueduct syndrome. Int J Pediatr Otorhinolaryngol. 2016;81:84-91.

2. Hartnick CJ, Kim HY, Chute PM, Parisier SC. Preventing labyrinthitis ossificans. Arch Otolaryngol Head Neck Surg. 2001;127(2):180
3. Berning AW, Arani K, Branstetter BF. Prevalence of superior semicircular canal dehiscence on high-resolution CT imaging in patients without vestibular or auditory abnormalities. AJNR Am J Neuroradiol. 2019;40(4):709-12.

4. Wieczorek SS, Anderson ME, Harris DA, Mikulec AA. Enlarged vestibular aqueduct syndrome mimicking otosclerosis in adults. Am J Otolaryngol. 2013;34(6):619-25.

5. Mori T, Westerberg BD, Atashband S, Kozak FK. Natural history of hearing loss in children with enlarged vestibular aqueduct syndrome. J Otolaryngol Head Neck Surg. 2008;37(1):112-8.

6. Saliba I, Gingras-Charland M-E, St-Cyr K, Décarie J-C. Coronal CT scan measurements and hearing evolution in enlarged vestibular aqueduct syndrome. Int $\mathrm{J}$ Pediatr Otorhinolaryngol. 2012;76(4):492-9.

7. Lin EP, Crane BT. The management and imaging of vestibular schwannomas. Am J Neuroradiol. 2017;38(11):2034-43.

8. Schwartz KM, Lane JI, Bolster BD, Neff BA. The utility of diffusion-weighted imaging for cholesteatoma evaluation. Am J Neuroradiol. 2011;32(3):430-6.

9. Alaani A, Chavda SV, Irving RM. The crucial role of imaging in determining the approach to glomus tympanicum tumours. Eur Arch Otorhinolaryngol. 2009;266(6):827-31.

10. Schmalfuss IM. Petrous apex. Neuroimaging Clin N Am. 2009;19(3):367-91.

11. Chapman PR, Shah R, Cure JK, Bag AK. Petrous apex lesions: pictorial review. Am J Roentgenol. 2011;196(3):WS26-37.

Open Access This chapter is licensed under the terms of the Creative Commons Attribution 4.0 International License (http://creativecommons. org/licenses/by/4.0/), which permits use, sharing, adaptation, distribution and reproduction in any medium or format, as long as you give appropriate credit to the original author(s) and the source, provide a link to the Creative Commons license and indicate if changes were made.

The images or other third party material in this chapter are included in the chapter's Creative Commons license, unless indicated otherwise in a credit line to the material. If material is not included in the chapter's Creative Commons license and your intended use is not permitted by statutory regulation or exceeds the permitted use, you will need to obtain permission directly from the copyright holder. 